\title{
Monocular Diplopia Associated with a Midbrain Stroke
}

\section{Santoshi Billakota and Larry B. Goldstein*}

Department of Neurology, Duke University Medical Center, Durham, North Carolina, USA

*Corresponding author: Santoshi Billakota, Duke University Medical Center PO Box 2905 27710, Durham, North Carolina, USA, Tel: (310) 699-1921; E-mail: santoshi.billakota@dm.duke.edu

Received October 15, 2014; Accepted December 16, 2014; Published December 19, 2014

Copyright: $\odot 2015$ Billakota S, et al. This is an open-access article distributed under the terms of the Creative Commons Attribution License, which permits unrestricted use, distribution, and reproduction in any medium, provided the original author and source are credited

\begin{abstract}
Background and Purpose

Monocular diplopia is often due to intraocular disease. It is rarely the result of cerebral causes, which include lesions in or near Brodmann's area nineteen, the frontal eye fields or the cerebellum. Monocular diplopia has not been reported in a patient with a midbrain lesion.

\section{Methods and Results}

A patient with a history of hypertension presented with right monocular diplopia and headache. There was no ocular cause of the patient's symptoms, and aside from bilateral ptosis, there were no relevant findings on neurological examination. MRI brain revealed an ischemic stroke affecting the right oculomotor nucleus. Her evaluation, including an ECG and 48-hour Holter monitor was unremarkable. A subsequent 30-day auto-trigger cardiac event monitor revealed paroxysmal atrial fibrillation.
\end{abstract}

\section{Conclusions}

This case shows that monocular diplopia can be associated with a midbrain lesion and underscores the importance of prolonged cardiac monitoring in patients with otherwise cryptogenic stroke.

Keywords: Monocular diplopia; Midbrain stroke; Atrial fibrillation

\section{Background}

Binocular diplopia resolves with covering either eye and is due to ocular misalignment. In contrast, monocular diplopia is limited to one eye and resolves only when the affected eye is covered. It can be nonphysiologic, caused by ocular pathology, and is rarely due to central causes.

\section{Case}

A 40-year old woman with a history of hypertension presented with light headedness, headache and blurred vision in her right eye. She had no history of LASIK surgery and was not psuedophakic. The patient described horizontal diplopia in her right eye that persisted on closure of the left eye and resolved with closure of the right eye. Examination revealed no visual field deficits and bilateral ptosis. There was no nystagmus, pursuit movements were smooth, pupillary light reflexes were normal, and there was no conjunctival injection or trauma. The reminder of her neurological examination was normal. An ophthalmology consultant found her visual acuity was 20/40 bilaterally with pinhole refraction and confirmed normal ocular motility and pupillary light responses. On slit lamp examination, there were no abnormalities of the cornea, anterior chamber or vitreous. The cup:disc ratio was 0.7 with normal intra-ocular pressures bilaterally, normal macula, mild arterio-venous nicking and no retinal lesions. MRI brain showed restricted diffusion in the right dorsomedial midbrain involving the oculomotor nerve nucleus (Figure 1). MR angiogram of her head and neck showed no steno-occlusive disease. ECG and cardiac telemetry monitoring were normal. A transesophageal echocardiogram showed no thrombus and a patent foramen ovale. An MR venogram of her pelvis and lower extremities was negative for deep vein thrombosis. Additional testing revealed a normal lipid panel, normal glycated hemoglobin and no evidence of a coagulation disorder or infectious etiology.

Given the distribution in a small penetrating vessel territory without other cause, the stroke was attributed to hypertensive disease. The patient began aspirin $81 \mathrm{mg}$ daily and chlorthalidone. A 48-hour Holter monitor obtained after hospital discharge was normal. A subsequent 30-day event-triggered cardiac monitor revealed paroxysmal atrial fibrillation (AF), atrial flutter, and possible supraventricular tachycardia. The patient was switched from aspirin to dabigatran.

\section{Discussion}

Monocular diplopia of physiological origin is most commonly due to periorbital causes including eyelid trauma and mass effect from a large growth such as a chalazion. Intraocular causes include irregularity of the Bowman's membrane, early keratoconus, lenticular changes, and retinal tears [2,3]. This patient had no ocular cause for her symptom.

Our patient initially had bilateral ptosis. The single, caudal third nerve subnucleus innervates the levator palpebrae muscles bilaterally, 
Page 2 of 2

and isolated, bilateral ptosis has been reported in a patient with a metastatic midbrain lesion [4,5]. As there was no pathological evaluation in our patient, it is possible that the tissue injury associated with the stroke may have been slightly more extensive than evident on MRI and could have affected the third nerve subnucleus, thereby causing bilateral ptosis.

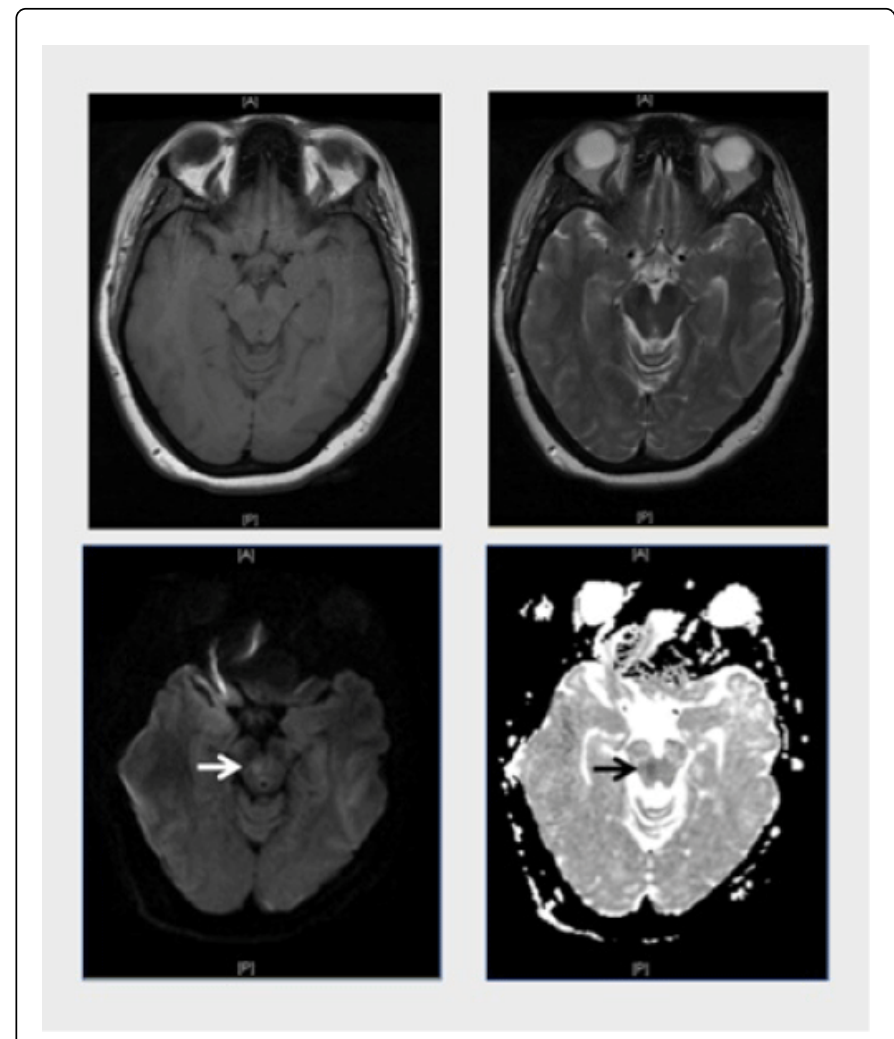

Figure 1: T1-weighted (upper left). T2-weighted (upper right), diffusion-weighted (lower left) and apparent diffusion coefficient (lower right) images of patient's MRI brain showing acute midbrain ischemic infarction (arrows).

Monocular diplopia due to a central cause is rare. Certain tumors (i.e., pituitary neoplasms) can produce a large scotoma caused by compression of macular nerve fibers that can lead to fixation errors, placing part of the image on the normally functioning macula and part on the macula corresponding to the compressed fibers, transmitting a displaced image. It can also occur in the setting of lesions that separate the receptor components of the occipital cortex or cause dissociative lesions between the frontal eye fields. Conjugate eye deviation due to cerebellar or vestibular lesions may produce episodic horizontal monocular diplopia [3].

A Medline search ([monocular diplopia.mp] [mesencephalon] and review of textbooks on neuro-ophthalmology and cerebrovascular disease did not identify previous reports of monocular diplopia associated with a midbrain lesion. Although the mechanism of our patient's monocular diplopia is uncertain, we speculate that it might have been the result of a change between the patient's eyelid position and the tear film interaction with her corneal surface [6]. Another possibility is that the midbrain injury led to a subtle exotropia that was not detected on bedside examination [7]. Unfortunately, further evaluation that may have provided additional information including degrees of separation between the images and the nature of the diplopia during near and far fixation is not available, and testing for an ocular tilt reaction and impairment of stereopsis was not performed.

A secondary point raised by this patient's case is that although ECGs, telemetry and an ambulatory 48-hour Holter monitor showed no arrhythmias, a subsequent 30-day event-triggered cardiac monitor revealed paroxysmal atrial fibrillation (AF). The EMBRACE trial assigned 572 subjects with recent cryptogenic ischemic stroke or TIA without known AF after a negative Holter monitor to wear an eventtriggered cardiac monitor for 30-days or a second 24-hour Holter monitor. New atrial fibrillation was found in $16.1 \%$ of subjects in the 30 -day monitoring group vs. $3.2 \%$ in the Holter group $(\mathrm{p}<0.001)$ [8]. Similarly, the retrospective Stroke and Monitoring for PAF in Real Time (SMART) Registry found that 30-day outpatient cardiac monitoring detected AF in $11 \%$ (26 of 236) of patients with cryptogenic ischemic stroke [9]. As with our patient, these studies show unrecognized paroxysmal AF is a potential cause for otherwise cryptogenic stroke.

\section{Conclusion}

Patients with monocular diplopia should have a thorough ophthalmologic evaluation and the possibility of a central etiology such as a midbrain lesion should be considered. Fitting of a refractive lenses and treatment of rare central causes will eliminate approximately $60 \%$ of all cases of monocular diplopia [2,3]. Prolonged cardiac monitoring should be pursued in patients with otherwise cryptogenic ischemic stroke.

\section{References}

1. Bender MB (1945) Polyopia and monocular diplopia of cerebral origin. Archives of Neurology 54: 323-338.

2. Records RE (1980) Monocular diplopia. Survey of Ophthalmology 24: 303-306.

3. Meadows JC (1973) Observations on a case of monocular diplopia of cerebral origin. Journal of Neurological Science 18: 249-253.

4. Brazis PW (1991) Localization of lesions of the oculomotor nerve: recent concepts. Mayo Clinic Proceedings 66: 1029-1035.

5. Kaski D, Ginsberg L (2014) Progressive bilateral ptosis in a patient with midbrain metastasis and chronic inflammatory demyelinating polyradiculoneuropathy. Practical Neurology 14: 349-350.

6. Ford J, Davis RM, Reed JW, Weaver RG, Craven TE, et al. (1997) Bilateral monocular diplopia associated with lid position during near work. Cornea 16: 525-530.

7. Ramachandran VS, Cobb S, Levi L (1994) The neural locus of binocular rivalry and monocular diplopia in intermittent exotropes. Neuro Report 5: 1141-1144.

8. Gladstone DJ, Spring M, Dorian P, Panzov V, Thorpe KE, et al. (2014) Atrial fibrillation in patients with cryptogenic stroke. New England Journal of Medicine 370: 2467-2477.

9. Flint AC, Nader M, Ren X, Rao V, Go A (2012) Detection of paroxysmal atrial fibrillation by 30 day event monitoring in cryptogenic ischemic stroke: the Stroke and Monitoring for PAF in Real Time (SMART) Registry. Stroke 10: 2788-2790 